\title{
Evaluación experimental de la eficiencia de un colector solar de tubos evacuados sin y con tubo de calor
}

Wilder Efraín Eufracio Arias*; Nathaly Ibeth Abregú Rodríguez; Dayana Rodríguez Espinoza.

Facultad de Ingeniería Química, Universidad Nacional del Centro del Perú (UNCP). Avenida Mariscal Castilla Nº 3909. Huancayo, Perú.

*E-mail: wilderefrain@yahoo.com

\begin{abstract}
Resumen
En la presente investigación se evaluó experimentalmente la eficiencia de un colector solar de tubos evacuados (CSTE) sin y con tubo de calor en días soleados, parcialmente nublados y nublados. En total se realizaron 46 experimentaciones durante 23 días: marzo a junio del 2017, con dos CSTE sin y con tubo de calor de 100 y 120 L, respectivamente. La radiación solar en la superficie horizontal y temperatura del agua en la salida del tanque de almacenamiento se registraron durante 5 horas (9:30 am a 2:30 pm) en intervalos de 30 y $10 \mathrm{~s}$, respectivamente. Para las representaciones y estimaciones de la eficiencia se utilizó el lenguaje de programación Matlab.

Los resultados muestran una ligera superioridad de eficiencia del CSTE con tubo de calor $(60,35 \%, 61,21 \%$ y $66,08 \%)$ comparado al sin tubo de calor $(57,17 \%, 57,29 \%$ y 59,56\%), medidos en días soleados, parcialmente nublados y nublados, respectivamente. Y se observa que en el CSTE con tubo de calor es mayor el incremento de la eficiencia en días nublados por las menores pérdidas de calor debido a un menor incremento de la temperatura, generando un mayor aprovechamiento de la energía solar incidente.

El análisis de varianza indicó que la condición de la radiación solar y tubo de calor del CSTE no influyen significativamente en la eficiencia energética de este colector solar.
\end{abstract}

De acuerdo a la condición de radiación solar (días soleados, parcialmente nublados y nublados), el CSTE con tubo de calor presentó una mayor eficiencia de 3,20\%, 3,92 \% y 6,52\%, respecto al CSTE sin tubo de calor.

Palabras claves: Energía Solar, Sistema de Calentamiento de Agua, Colector Solar de Tubos Evacuados, Tubo de Calor, Eficiencia

\section{Experimental evaluation of the efficiency of a solar tube collector evacuated with and without heat pipe}

\begin{abstract}
This research study evaluated the efficiency of a solar evacuated tube collector (SETC) with and without a heat pipe on sunny, partially cloudy and cloudy days, experimentally. A total of 46 experiments were conducted during a period of 23 days: from March to June 2017 with two (SETC) with and without a heat pipe of 100 and 120 L, respectively. Solar radiation in the horizontal surface, and water temperature at the outlet of the storage tank were registered for 5 hours $(9: 30$ a.m to 2:30 p.m) with intervals of 30 and 10 seconds, respectively. The Matlab programming language was used for representations and estimates of efficiency.

Results show a slight superiority in the efficiency of the SETC with a heat pipe of $(60,35 \%, 61,21 \%$ and $66,08 \%)$ compared to the one without heat pipe $(57,17 \%, 57,29 \%$ and $59,56 \%)$, measured on sunny, partially cloudy and cloudy days, respectively. It was observed that on cloudy days, the SETC with a heat pipe presented an increase in the efficiency as a result of lower heat losses due to a lower increase in temperature thus generating a greater use of incident solar energy.

The variance analysis indicated that the solar radiation and tube heat condition of the SETC do not influence significatively in the energy efficiency of this solar collector. According to the condition of solar radiation (sunny, partially cloudy and cloudy days), the SETC with a heat pipe presented a higher efficiency of $3.20 \%, 3.92 \%$, and $6.52 \%$, compared to the SETC without a heat pipe.
\end{abstract}

Key words: solar energy, water heating system, solar evacuated tube collector, heat pipe, efficiency.

Cita: Eufracio Arias, W. E., Abregú Rodríguez, N. I. y Rodríguez Espinoza D. (2019). Evaluación experimental de la eficiencia de un colector solar de tubos evacuados sin y con tubo de calor. Revista Fuentes: El reventón energético, vol 17(1), 7-17. 


\section{Introducción}

Desde tiempos remotos, los diferentes recursos no renovables (fósiles) han sido utilizados por el hombre como fuentes de energía. Actualmente la demanda de energía se incrementa a medida que la población y el consumo per cápita crecen, tanto así que los recursos energéticos como el petróleo y sus derivados se consumen en forma exponencial y por ende se liberan considerables cantidades de gases tóxicos a la atmósfera (CO, NOx, SOx, etc.), estos gases pueden producir efectos negativos al medio ambiente (efecto invernadero) y salud humana (Menjura et al, 2011; Forero et al, 2012).

Estos fenómenos naturales se intensifican afectando poblaciones, desapareciendo especies animales y vegetales a causa del daño en los ecosistemas, haciendo que el planeta se convierta cada vez menos habitable para los humanos.

En los últimos años, en el mundo se incrementó el interés por la utilización de energías renovables o alternativas debido a que son inagotables y limpias ambientalmente. Entre las no convencionales tenemos a la energía solar, eólica, de biomasa, geotérmica y mareomotriz (Vidal y Fontalvo, 2018; Amaris et al, 2015; Ayala et al, 2011). De ellas, la energía solar es la más potencial debido a que puede sustituir 6720 veces más la necesidad energética del mundo durante un año.

Perú es un país privilegiado con este recurso debido a su ubicación geográfica (latitud). Generalmente la intensidad de radiación solar es alta en gran parte del día, durante los días del año y no presenta variaciones considerables. Sin embargo, a pesar de disponer de ésta energía, no se genera tecnología y se prefiere importar equipos de otros países como China. Los colectores solares los cuales muchas veces no cumplen con las especificaciones de diseño requeridas para obtener un desempeño optimo en otro lugar con diferente latitud y condiciones ambientales son un ejemplo de esto.

En el mercado se comercializan diferentes colectores solares de tubos evacuados sin y con tubo de calor con diferentes características y precios, el usuario no conoce las ventajas y desventajas de cada uno de ellos para decidir por cual optar. Frente a ello es necesario estudiar el comportamiento de estos colectores a condiciones reales de operación, por lo que en la investigación se evaluó la eficiencia de un CSTE sin y con tubo de calor en la Facultad de Ingeniería Química de la Universidad Nacional del Centro del Perú.

\section{Radiación solar}

La radiación solar tiene su origen en el Sol, que es una esfera gaseosa de $1391000 \mathrm{~km}$ de diámetro, una de las más de 135000 millones de estrellas de la Vía Láctea. El Sol es un inmenso reactor de fusión termonuclear que quema cada segundo $6 \times 10^{8} \mathrm{TM}$ de hidrógeno a $2 \times 10^{7} \mathrm{~K}$. De la radiación solar sólo llega un $51,6 \%$ a la superficie de la tierra. Los restantes $48,4 \%$ se pierde como reflexión $(31 \%)$ y absorción $(17,4 \%)$ en la capa de aire. (Larrea \& Bayas, 2011, p. 2).

La radiación solar extraterrestre varía por dos fuentes, la primera es la variación en la radiación emitida por el sol y la segunda es la variación de la distancia de la tierra al sol, sin embargo, genera una variación del flujo de la radiación extraterrestre en el rango de $\pm 3,3 \%$. (Duffie \& Beckman, 2013, p. 6)

La energía solar presenta dos características que la diferencian de las fuentes energéticas convencionales: dispersión e intermitencia.

Es necesario diferenciar los tipos y terminologías de la radiación solar los cuales son:

- Radiación directa: Se recibe directamente del Sol.

- Radiación difusa: Se recibe del Sol después de ser desviada por dispersión atmosférica.

- Radiación reflejada o terrestre: Proviene del reflejo de objetos terrestres.

- Radiación total: Es la suma de las radiaciones directa, difusa y reflejada.

- Radiación global: Es la suma de las radiaciones directa y difusa. Esta radiación se utiliza para el cálculo de colectores solares.(Loayza, 2012).

Las razones de aprovechar la energía solar se debe a que los combustibles fósiles no son ambientalmente amigables y emiten una cantidad significativa de contaminantes causando serios asuntos medioambientales como el calentamiento global, la reducción drástica de la capa de ozono y el cambio climático. Las fuentes renovables de energía, con ventajas de ser ambientalmente amigables y abundantes en su disponibilidad, son la opción prometedora para responsabilizarse de la demanda creciente de energía en todo el mundo. (Park, Pandey, Tyagi, \& Tyagi, 2014, p. 106) 
Entre estas fuentes renovables, la energía solar ha recibido una atención considerable debido a su facilidad de acceso y alta potencialidad para generar electricidad y calor (Jafarkazemi \& Ahmadifard, 2013, pág. 55). Dentro de sus aplicaciones, puede ser ampliamente utilizada para el calentamiento de agua en sistemas de agua caliente, piscinas, así como también fuentes de energía de soporte para instalaciones de calefacción. La energía de la radiación solar se convierte a calor con colectores solares. (Zima \& Dziewa, 2010, p. 47).

\section{Principales técnicas de conversión de la radiación solar a térmica}

Las principales tecnologías son:

a) Circulación natural (termosifón). No hay elementos en el sistema del tipo electromecánico.

Un calentador solar de agua con termosifón típico consiste de un colector y un tanque de almacenamiento conectado por un tubo de salida y uno de retorno. El tanque de almacenamiento se instala por encima del colector para permitir la circulación natural durante el día y prevenir la circulación inversa por la noche. El flujo del termosifón en el circuito del colector se produce por la diferencia del peso entre la columna del fluido caliente en el tubo de retorno y los tubos del colector y la columna del fluido frío en el tubo de salida. (Budihardjo \& Morrison, 2009, p. 50-51)

Esta tecnología presenta dos limitaciones principales. No son útiles donde la temperatura ambiente cae debajo del punto de congelación y la segunda tiene que ver con el ensuciamiento en los tubos de diámetro pequeño del colector. (Taherian, Rezania, Sadeghi, \& Ganji, 2011, p. 302)

b) Circulación forzada. El fluido circula gracias a una bomba de circulación.

\section{Colector solar de tubos evacuados}

Estos colectores se utilizan en aplicaciones que requieren temperaturas moderadas entre 50 y $95^{\circ} \mathrm{C}, \mathrm{y} / \mathrm{o}$ en climas muy fríos.

La eficiencia térmica de estos colectores es superior a la de los tradicionales paneles planos, debido a que, en estos últimos, la mayor pérdida de calor se produce por conducción y convección entre la superficie captadora y el cristal exterior a través del aire existente entre ellos. El vacío que lo caracteriza contribuye también a minimizar la influencia de las condiciones climáticas sobre los materiales empleados, evitando su rápido deterioro y mejorando así la durabilidad y el rendimiento global del sistema. (Salcedo, 2011, p. 31)

Los colectores de tubos de vacío están construidos de un número de tubos de vidrio. Cada tubo está hecho de vidrio recocido y tiene una placa de absorción dentro del tubo, porque el tubo es la configuración natural de un colector evacuado. A fin de reducir las pérdidas de calor por conducción y convección, durante el proceso de fabricación, se crea un vacío dentro del tubo de vidrio. El único mecanismo de pérdida de calor restante es la radiación. La ausencia de aire en el tubo crea un excelente aislamiento, lo que permite que deban alcanzarse temperaturas más altas en la placa de absorción. A fin de mejorar la eficiencia del colector de tubos de vacío hay varios tipos de concentradores dependiendo de su radio cóncavo. En todos ellos se utiliza el recubrimiento selectivo del absorbedor. (Jesko, 2008, p. 25)

En el mercado se comercializa los siguientes colectores de tubos evacuados:

\section{Colector solar de flujo directo}

Estos consisten en un grupo de tubos de vidrio dentro de cada uno de los cuales hay una aleta de aluminio absorbedora, conectada a un tubo de metal (normalmente cobre) o tubo de vidrio. La aleta posee un recubrimiento selectivo que absorbe la radiación solar, e inhibe la pérdida de calor radiactivo.

El fluido de transferencia de calor es el agua y se distribuye a través de las tuberías, una para la entrada del líquido y el otro para la salida de fluidos. Los colectores de tubos de vacío de corriente directa vienen en varias variedades de acuerdo al tipo de tubería utilizada.

1. Fluido concéntrico de entrada y salida (vidriometal). Estos utilizan un único tubo de vidrio. Dentro de este está la tubería de cobre adosada a la aleta.

2. Tuberías de entrada y salida separadas (vidriometal). Este es el tipo tradicional de colectores de tubos de vacío.

3. Dos tubos de vidrio fundido juntos en un extremo (vidrio-vidrio). (Conicet, 2009)

En el mercado un CSTE no contienen aletas dentro del tubo. 


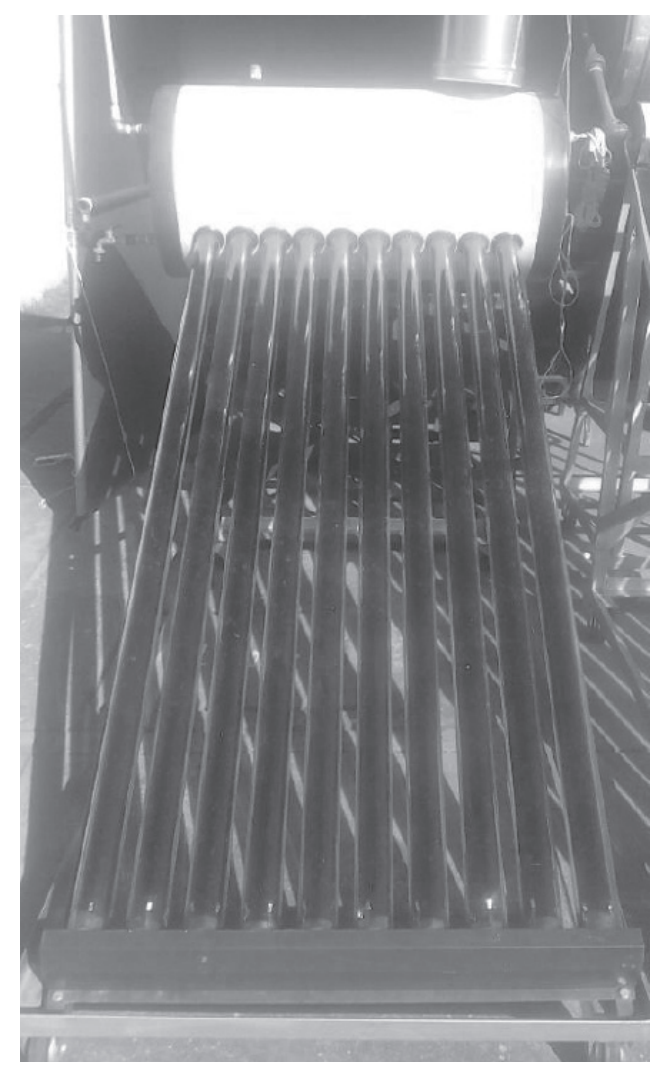

Figura 1. CSTE de flujo directo.

\section{Colector solar con tubo de calor (heat pipe)}

En este sistema los tubos de vacío llevan un fluido vaporizante que no puede salir del interior del tubo y que funciona como caloportador. Este fluido se evapora por efecto de la radiación solar, asciende hasta el extremo superior del tubo que se encuentra a temperatura inferior, esto hace que el vapor se condense, ceda su energía y retorne a su estado líquido cayendo por acción de la gravedad a la parte inferior del tubo, donde al recibir más radiación, vuelve a evaporarse y comienza un nuevo ciclo. (Conicet, 2009, p. 5)

Una ventaja del sistema de tubos de calor sobre el de flujo directo es la conexión "seca" entre el absorbedor y la cabecera, lo que hace más fácil la instalación y el cambio de los tubos, ya que este puede hacerse sin vaciar el fluido de todo el sistema. Un inconveniente de estos colectores con tubos de calor es que deben ser montados con un ángulo mínimo de inclinación de alrededor de $25^{\circ}$ con el fin de permitir que el fluido interno de la tubería de calor retorne a la zona de absorción de calor, en cambio los de flujo directo pueden ser instalados de manera horizontal. (Martinez, Franco, \& Alonso, 2013, p. 24)

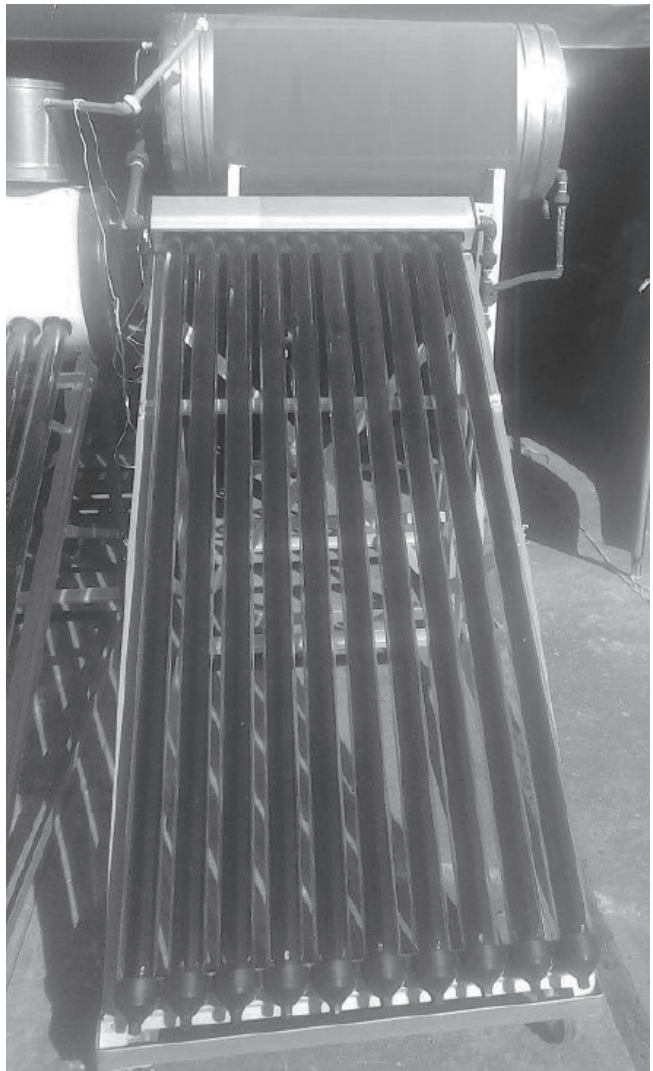

Figura 2. CSTE con tubo de calor (heat pipe).

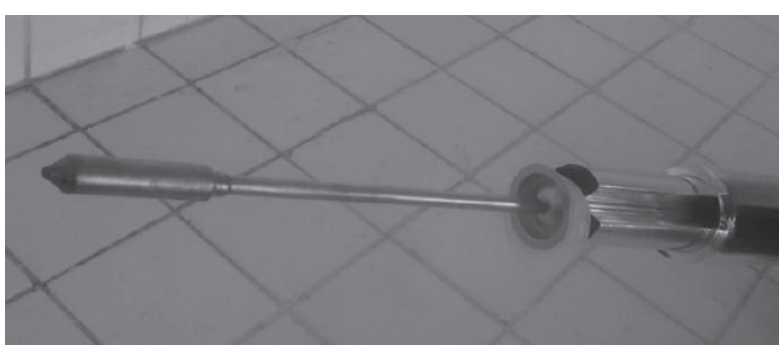

Figura 3. Cabezal del tubo de calor.

\section{Orientación e inclinación}

Los colectores solares generalmente son instalados en una posición fija, por lo que su ángulo de inclinación debe ser óptimo. La orientación está definida por su ángulo de azimut e inclinación.

El ángulo de inclinación de cualquier colector es el ángulo entre el plano de la superficie del colector y la horizontal, es óptimo cuando el ángulo de incidencia es mínimo para que la radiación directa sea la máxima. (Skeiker, 2009, p. 2440)

Además, este es uno de los parámetros importantes para captar la máxima radiación solar que incide en 
los paneles solares. Este ángulo es específico para cada lugar y depende del día, mes y año por la posición del Sol. (Kumar \& Chandel, 2013, p. 503)

Los colectores se orientan al sur en el hemisferio norte $\mathrm{y}$ al norte en el sur y depende de tres condiciones de utilización, que son:

- El valor de la latitud del lugar para instalaciones de utilización en todas las estaciones del año.

- $\quad+10^{\circ}$ respecto de lo anterior, si es sólo durante el invierno.

- $-10^{\circ}$ si la utilización principal se produce sólo durante el verano.

Actualmente, se realizan diversas investigaciones para la determinación del ángulo de inclinación y azimut óptimo, estas varían de lugar de estudio, tipo de colector solar y características del modelo matemático utilizado. Prácticamente todas estas investigaciones se enfocaron para lugares del continente asiático o europeo. La determinación exacta del ángulo de inclinación para cada lugar de interés es esencial para la producción de energía máxima por el sistema.

\section{Evaluación experimental del colector solar}

Principalmente hay dos métodos generales de prueba que se siguen en el análisis del rendimiento (eficiencia) del colector solar de placa plana: prueba estacionaria (interno) y dinámico (externo) del colector solar. Los modelos dinámicos se basaron inicialmente en un modelo de un sólo nodo. El modelo de un nodo se actualizó, el modelo multinodos se introdujo, considerando que el colector consiste en múltiples nodos, cada uno con una sola temperatura y capacitancia. La suposición de las condiciones de estado estacionario (temperatura de entrada, razón de flujo del fluido en los tubos, irradiancia incidente y condiciones ambientales constante) en los métodos estacionarios hacen las pruebas del colector más complicadas y caras. Desde que los colectores solares normalmente operan en condiciones transientes (al aire libre), las condiciones de prueba de estado estacionario no son típicos de la operación normal. (Ahmad, 2012, p. 9-10).

El método de prueba en estado estacionario es el procedimiento usual adoptado por la mayoría de estándares comunes. Las pruebas interiores pueden ser realizadas en laboratorios certificados con un simulador solar, mientras que los procedimientos externos requieren condiciones de ambientes específicos (datos climáticos y de medio ambiente). Pero, hay discrepancias frecuentes entre los resultados internos y externos, probablemente debido a los errores en describir la temperatura del cielo en las pruebas internas. (Tagliafico, Scarpa, \& De Rosa, 2014, pág. 533). Por ello, el estudio transiente de los colectores es de gran interés por su uso en predecir el comportamiento dependiente del tiempo del colector a intensidad variable de la radiación solar y condiciones meteorológicas. (Hamed, Fellah, \& Ben Brahim, 2014, p. 939)

Las pruebas dinámicas son más fáciles de realizar experimentalmente y requieren menos inversión, pero el modelo y cálculos serán más complejos. Sin embargo, el modelo y cálculo se deben desarrollar una sola vez, pero los gastos para el experimento son para cada prueba.

\section{Metodología}

\section{Materiales, equipos e instrumentos}

Los materiales que se utilizaron fueron: manqueras de $1 / 2$ pulg de $20 \mathrm{~m}$, extensión de $20 \mathrm{~m}, 5$ codos de CPVC de $1 / 2$ ", 1 llave de paso de metal $1 / 2$ ", 3 unión universal de CPVC de 1/2", 7 niple de CPVC de $1 / 2$ ", 1 "T" de CPVC de $1 / 2$ ", $3 \mathrm{~m}$ de tubo de CPVC de $1 / 2$ " y teflón.

Mientras que los equipos e instrumentos que se utilizaron fueron: un sistema de recolección de datos-usb de marca Inducontrol y modelo usb-log, cuatro sensores de temperaturas de modelo DT029 cuya precisión es $\pm 2 \%$ del rango total y resolución de $0,03{ }^{\circ} \mathrm{C}$, un solarímetro marca Kimo y modelo SL 100 cuya precisión es $1 \mathrm{~W} / \mathrm{m}^{2}$ y rango de 1 a $1300 \mathrm{~W} / \mathrm{m}^{2}$, un CSTE sin tubos de calor, un CSTE con tubos de calor y una laptop.

\section{Procedimiento}

\section{Para la recolección de datos se procedió a:}

- Ubicar el solarímetro SL100 (posición horizontal) y los colectores solares con y sin tubo de calor en un lugar donde los rayos solares inciden directamente y no se produzcan sombras sobre el instrumento o equipos durante la experimentación.

- Llenar con agua potable los colectores solares y tanques de almacenamientos.

- Instalar los sensores de temperatura en la parte superior de los tanques de almacenamiento de los colectores solares (al nivel de salida del agua caliente), dos sensores para cada colector solar. Luego conectar los sensores al recolector de datos y a la laptop. 
- Activar el programa Multilab para la recolección de datos de las temperaturas.

- Monitorear los datos de temperatura del agua de los colectores y radiación solar en horizontal, en intervalos de 10 y $30 \mathrm{~s}$, respectivamente.

- Registrar la irradiancia solar en la horizontal cada $30 \mathrm{~s}$.

- Realizar el registro de datos durante 5 horas, desde las 9:30 am hasta las 2:30 pm.

- Concluidas las mediciones, descargar el agua de los colectores solares.

- Exportar los datos del programa Multilab al Microsoft Excel.

Para las estimaciones de la eficiencia se utilizó la siguiente ecuación:

$$
\eta=\frac{\text { Energía o calor útil }\left(\mathrm{E}_{\mathrm{T}}\right)}{\text { Energía solar incidente total }\left(\mathrm{E}_{\mathrm{S}}\right)}
$$

La energía o calor útil del colector solar, se calculó a partir de la ecuación:

$$
\mathrm{E}_{\mathrm{T}}=\mathrm{mC}_{\mathrm{P}} \Delta \mathrm{T}
$$

Donde $\mathrm{m}$ es la masa del agua almacenada en el colector solar y tanque de almacenamiento, $\mathrm{C}_{\mathrm{P}}$ es calor especifico del agua y $\Delta \mathrm{T}$ el incremento de la temperatura del agua.

Mientras que la energía solar incidente total está dada por:

$$
\mathrm{E}_{\mathrm{S}}=\mathrm{GAt}
$$

Donde $\mathrm{G}$ es la irradiancia solar en la horizontal, A es el área de absorción o apertura del CSTE y t es el intervalo de tiempo de las mediciones.

\section{Diseño de investigación}

El diseño de la investigación es experimental factorial simple, con dos factores, dos niveles y 10 réplicas.

$$
\mathrm{N}=2^{2} \times 10=40
$$

Las variables de la investigación son:

Variable independiente:

- Condición de la radiación solar:

- Día soleado.

- Día parcialmente nublado.

- Tipo de colector solar de tubos evacuados

- CSTE sin tubo de calor.

- CSTE con tubo de calor.
Variable dependiente:

- Eficiencia (\%)

Adicionalmente, en la investigación se experimentó en días nublados para realizar las respectivas comparaciones, el detalle que sólo fueron 3 días.

\begin{tabular}{|c|c|c|c|c|}
\hline $\mathbf{N}^{\circ}$ & Día & $\mathrm{C} / \mathrm{RS}^{\mathrm{a}}$ & \multicolumn{2}{|c|}{ CSTE } \\
\hline 1 & $15 / 03 / 2017$ & S & $\mathrm{C} / \mathrm{TC}$ & $\mathrm{S} / \mathrm{TC}$ \\
\hline 2 & $16 / 03 / 2017$ & $S$ & $\mathrm{C} / \mathrm{TC}$ & $\mathrm{S} / \mathrm{TC}$ \\
\hline 3 & $21 / 03 / 2017$ & $S$ & $\mathrm{C} / \mathrm{TC}$ & $\mathrm{S} / \mathrm{TC}$ \\
\hline 4 & $22 / 03 / 2017$ & $S$ & $\mathrm{C} / \mathrm{TC}$ & $\mathrm{S} / \mathrm{TC}$ \\
\hline 5 & $26 / 03 / 2017$ & S & $\mathrm{C} / \mathrm{TC}$ & $\mathrm{S} / \mathrm{TC}$ \\
\hline 6 & $24 / 05 / 2017$ & S & $\mathrm{C} / \mathrm{TC}$ & $\mathrm{S} / \mathrm{TC}$ \\
\hline 7 & $31 / 05 / 2017$ & $\mathrm{~S}$ & $\mathrm{C} / \mathrm{TC}$ & $\mathrm{S} / \mathrm{TC}$ \\
\hline 8 & $01 / 06 / 2017$ & $\mathrm{~S}$ & $\mathrm{C} / \mathrm{TC}$ & $\mathrm{S} / \mathrm{TC}$ \\
\hline 9 & $07 / 06 / 2017$ & $S$ & $\mathrm{C} / \mathrm{TC}$ & $\mathrm{S} / \mathrm{TC}$ \\
\hline 10 & $08 / 06 / 2017$ & $S$ & $\mathrm{C} / \mathrm{TC}$ & $\mathrm{S} / \mathrm{TC}$ \\
\hline 11 & $13 / 03 / 2017$ & $\mathrm{PN}$ & $\mathrm{C} / \mathrm{TC}$ & $\mathrm{S} / \mathrm{TC}$ \\
\hline 12 & $20 / 03 / 2017$ & $\mathrm{PN}$ & $\mathrm{C} / \mathrm{TC}$ & $\mathrm{S} / \mathrm{TC}$ \\
\hline 13 & $23 / 03 / 2017$ & PN & $\mathrm{C} / \mathrm{TC}$ & $\mathrm{S} / \mathrm{TC}$ \\
\hline 14 & $25 / 03 / 2017$ & $\mathrm{PN}$ & $\mathrm{C} / \mathrm{TC}$ & $\mathrm{S} / \mathrm{TC}$ \\
\hline 15 & $26 / 05 / 2017$ & PN & $\mathrm{C} / \mathrm{TC}$ & $\mathrm{S} / \mathrm{TC}$ \\
\hline 16 & $30 / 05 / 2017$ & $\mathrm{PN}$ & $\mathrm{C} / \mathrm{TC}$ & $\mathrm{S} / \mathrm{TC}$ \\
\hline 17 & $02 / 05 / 2017$ & PN & $\mathrm{C} / \mathrm{TC}$ & $\mathrm{S} / \mathrm{TC}$ \\
\hline 18 & $03 / 05 / 2017$ & $\mathrm{PN}$ & $\mathrm{C} / \mathrm{TC}$ & $\mathrm{S} / \mathrm{TC}$ \\
\hline 19 & $05 / 05 / 2017$ & $\mathrm{PN}$ & $\mathrm{C} / \mathrm{TC}$ & $\mathrm{S} / \mathrm{TC}$ \\
\hline 20 & 06/05/2017 & $\mathrm{PN}$ & $\mathrm{C} / \mathrm{TC}$ & $\mathrm{S} / \mathrm{TC}$ \\
\hline 21 & $10 / 03 / 2017$ & $\mathrm{~N}$ & $\mathrm{C} / \mathrm{TC}$ & $\mathrm{S} / \mathrm{TC}$ \\
\hline 22 & $14 / 03 / 2017$ & $\mathrm{~N}$ & $\mathrm{C} / \mathrm{TC}$ & $\mathrm{S} / \mathrm{TC}$ \\
\hline 23 & $24 / 03 / 2017$ & $\mathrm{~N}$ & $\mathrm{C} / \mathrm{TC}$ & $\mathrm{S} / \mathrm{TC}$ \\
\hline
\end{tabular}

En la siguiente tabla, se presenta la información de las experimentaciones que se realizaron.

Tabla 1. Experimentaciones

${ }^{\mathrm{a}}$ Condición de la radiación solar.

$\mathrm{S}$ : Día soleado, PN: Día parcialmente nublado, N: Día nublado, C/TC: Con tubo de calor y S/TC: Sin tubo de calor.

\section{Resultados y discusión}

\section{Características de los colectores solares de tubos evacuados sin y con tubo de calor}

Las características del CSTE que influyen en el rendimiento y funcionamiento son el área de apertura y la capacidad del tanque de almacenamiento. Respecto al primero la diferencia es $0,01 \mathrm{~m}^{2}$; por lo que no influye en los resultados de la investigación, mientras que la diferencia en la capacidad de los colectores es 20 L, según la literatura, cuando las variaciones no son considerables, el efecto de esta diferencia tampoco es 
significativo. Las temperaturas del agua en el tanque de almacenamiento son superiores en el colector sin tubo de calor porque su capacidad es $100 \mathrm{~L}$, respecto al colector con tubo de calor que es $120 \mathrm{~L}$. Esto se debe a que los calores útiles son similares, pero la diferencia de la masa de agua del colector genera que uno se caliente más que el otro.

\section{Comportamiento de la temperatura del agua en función a la radiación solar}

En las siguientes figuras se presentan el comportamiento de la radiación solar en la horizontal y la temperatura del agua en el tanque de almacenamiento del CSTE sin y con tubo de calor del en un día soleado (24/05/2017).

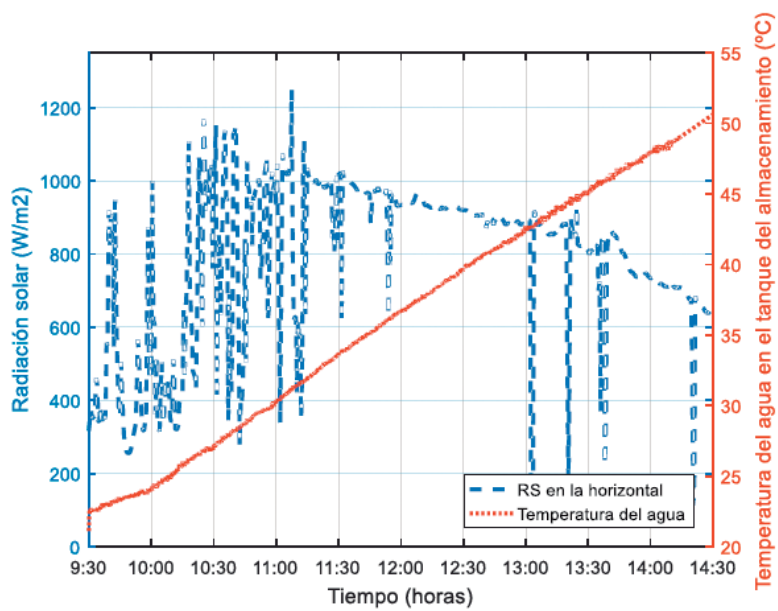

Figura 4. Radiación solar y temperatura del agua del CSTE sin tubo de calor de un día soleado (24/05/2017)

En un día soleado, la irradiancia solar promedio es 798 $\mathrm{W} / \mathrm{m}^{2}$ y el incremento de la temperatura del agua varía entre 21,14 y $29,50^{\circ} \mathrm{C}$; y 18,51 y $25,69^{\circ} \mathrm{C}$ en un CSTE $\sin \mathrm{y}$ con tubo de calor, respectivamente.

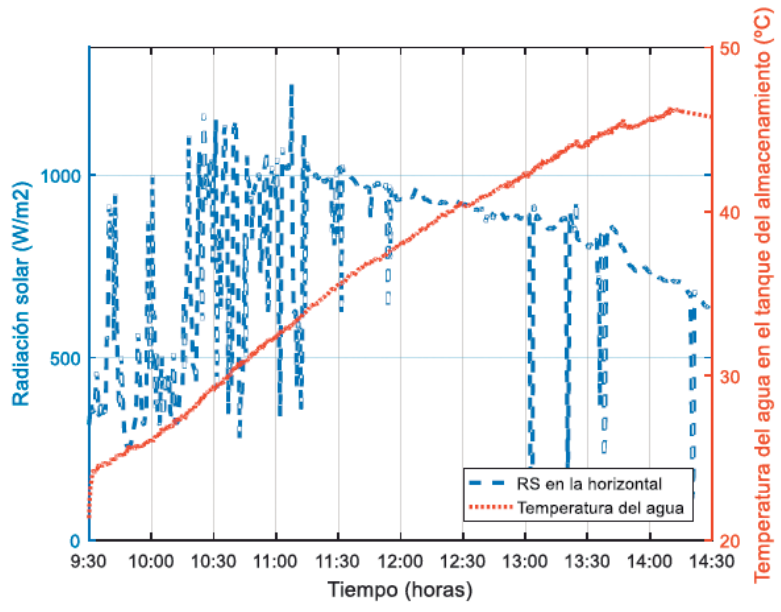

Figura 5. Radiación solar y temperatura del agua del CSTE con tubo de calor de un día soleado (24/05/2017).
Ahora se presenta la misma información para ambos colectores, pero para un día parcialmente nublado, las mediciones corresponden al día 30/05/2017.

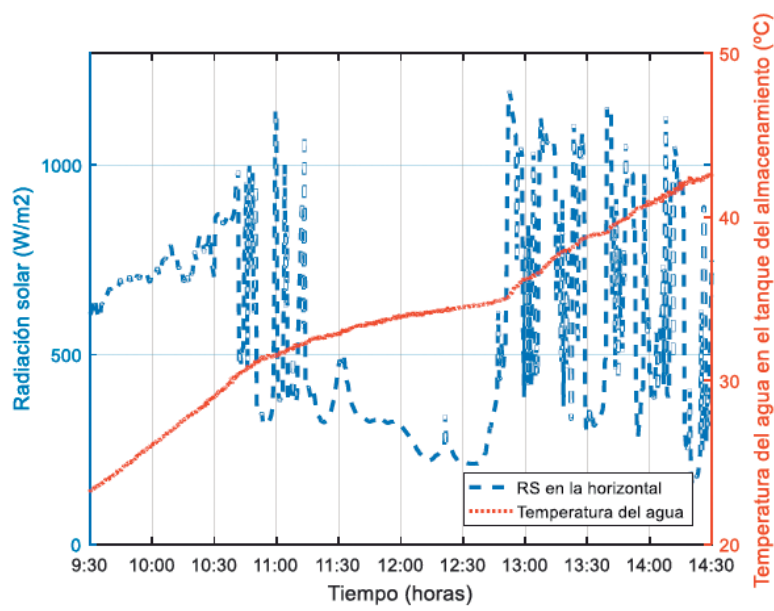

Figura 6. Radiación solar y temperatura del agua del CSTE sin tubo de calor de un día parcialmente nublado $(30 / 05 / 2017)$

En un día parcialmente nublado, el promedio de la irradiancia solar es $683 \mathrm{~W} / \mathrm{m}^{2}$ y el incremento de la temperatura del agua oscila entre 17,41 y $26,44{ }^{\circ} \mathrm{C}$; y 15,42 y $25,28^{\circ} \mathrm{C}$ en un CSTE sin y con tubo de calor, respectivamente.

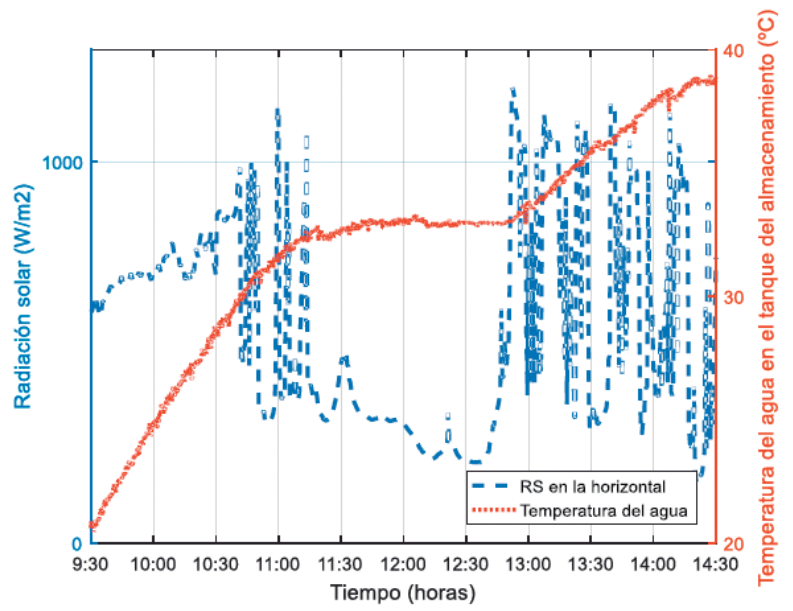

Figura 7. Radiación solar y temperatura del agua del CSTE con tubo de calor de un día parcialmente nublado $(30 / 05 / 2017)$

Finalmente, se muestra las mediciones de un día nublado $(14 / 03 / 2017)$. 


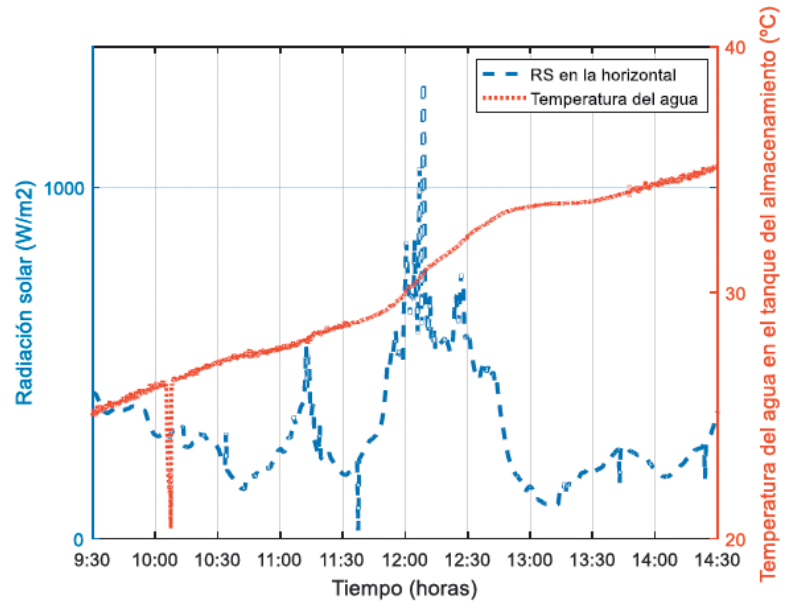

Figura 8. Radiación solar y temperatura del agua del CSTE sin tubo de calor de un día nublado (14/03/2017)

Finalmente, para un día nublado, el promedio de la irradiancia solar es $334 \mathrm{~W} / \mathrm{m}^{2}$ y el incremento de la temperatura del agua varía entre 8,20 y $15,37{ }^{\circ} \mathrm{C}$; y 7,64 y $15,37^{\circ} \mathrm{C}$ en un CSTE sin y con tubo de calor, respectivamente.

El incremento de la temperatura del agua tiene relación directa con la irradiancia solar; a mayor radiación solar mayor temperatura, pero no es proporcional, porque a mayor radiación se incrementa las pérdidas de calor en los colectores solares. El incremento de la temperatura en el colector con tubo de calor es menor porque la capacidad es mayor en $20 \mathrm{~L}$, respecto al sin tubo de calor.

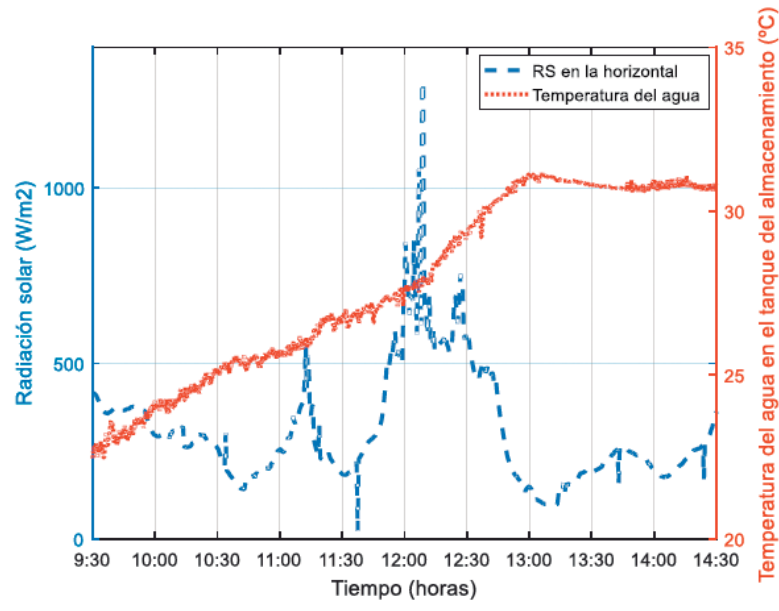

Figura 9. Radiación solar y temperatura del agua del CSTE con tubo de calor de un día nublado (14/03/2017)

\section{Eficiencia del CSTE sin y con tubo de calor}

A continuación, se presenta los promedios de las mediciones realizadas en las experimentaciones con el CSTE sin y con tubo de calor.

Tabla 2. Radiación solar y temperatura del agua promedio en el CSTE sin tubo de calor

\begin{tabular}{|c|c|c|c|c|c|}
\hline \multirow{2}{*}{$\mathbf{N}^{\circ}$} & \multirow{2}{*}{ Día } & \multirow{2}{*}{$\begin{array}{c}\text { C/ } \\
\mathbf{R S}^{\mathbf{a}}\end{array}$} & \multirow{2}{*}{$\begin{array}{c}\mathbf{R S}^{\mathbf{b}} \\
\left(\mathbf{W} / \mathbf{m}^{2}\right)\end{array}$} & \multicolumn{2}{|c|}{$\begin{array}{c}T_{\mathrm{a}}^{\mathrm{c}} \\
\left({ }^{\circ} \mathrm{C}\right)\end{array}$} \\
\hline & & & & $\begin{array}{c}\mathrm{CS} \mathrm{S} / \\
\mathrm{TC}^{\mathrm{d}}\end{array}$ & $\mathrm{CS} \mathrm{C} / \mathrm{TC}^{\mathrm{e}}$ \\
\hline 1 & $15 / 03 / 17$ & S & 774,38 & 35,63 & 35,63 \\
\hline 2 & $16 / 03 / 17$ & S & 914,74 & 36,56 & 36,56 \\
\hline 3 & $21 / 03 / 17$ & S & 884,24 & 40,65 & 40,65 \\
\hline 4 & $22 / 03 / 17$ & S & 785,54 & 39,19 & 39,19 \\
\hline 5 & $26 / 03 / 17$ & S & 838,22 & 39,23 & 39,23 \\
\hline 6 & $24 / 05 / 17$ & S & 781,04 & 36,35 & 36,35 \\
\hline 7 & $31 / 05 / 17$ & S & 744,99 & 37,59 & 37,59 \\
\hline 8 & $01 / 06 / 17$ & S & 682,50 & 37,87 & 37,87 \\
\hline 9 & $07 / 06 / 17$ & S & 775,78 & 38,77 & 38,77 \\
\hline 10 & $08 / 06 / 17$ & S & 800,28 & 38,94 & 38,94 \\
\hline 11 & $13 / 03 / 17$ & PN & 731,41 & 32,78 & 32,78 \\
\hline 12 & $20 / 03 / 17$ & $\mathrm{PN}$ & 921,12 & 37,02 & 37,02 \\
\hline 13 & $23 / 03 / 17$ & $\mathrm{PN}$ & 621,70 & 36,10 & 36,10 \\
\hline 14 & $25 / 03 / 17$ & PN & 639,25 & 34,51 & 34,51 \\
\hline 15 & $26 / 05 / 17$ & $\mathrm{PN}$ & 610,09 & 37,36 & 37,36 \\
\hline 16 & $30 / 05 / 17$ & $\mathrm{PN}$ & 563,84 & 33,66 & 33,66 \\
\hline 17 & $02 / 06 / 17$ & $\mathrm{PN}$ & 706,31 & 36,47 & 36,47 \\
\hline 18 & $03 / 06 / 17$ & $\mathrm{PN}$ & 671,96 & 37,20 & 37,20 \\
\hline 19 & $05 / 06 / 17$ & $\mathrm{PN}$ & 685,78 & 36,11 & 36,11 \\
\hline 20 & $06 / 06 / 17$ & PN & 679,93 & 35,98 & 35,98 \\
\hline 21 & $10 / 03 / 17$ & $\mathrm{~N}$ & 226,74 & 27,25 & 27,25 \\
\hline 22 & $14 / 03 / 17$ & $\mathrm{~N}$ & 308,93 & 30,34 & 30,34 \\
\hline 23 & $24 / 03 / 17$ & $\mathrm{~N}$ & 466,57 & 31,44 & 31,44 \\
\hline
\end{tabular}

a Condición de la radiación solar, ${ }^{\mathrm{b}}$ Irradiancia solar en la horizontal promedio, ${ }^{\mathrm{c}}$ Temperatura del agua promedio del tanque de almacenamiento del CSTE, ${ }^{\mathrm{d}}$ CSTE sin tubo de calor, ${ }^{\mathrm{e}} \mathrm{CSTE}$ con tubo de calor.

En las Tabla 3 y Tabla 4 se presentan la información necesaria para la estimación de la eficiencia del CSTE sin y con tubo de calor, respectivamente: 
Tabla 3. Eficiencia del CSTE sin tubo de calor

\begin{tabular}{|c|c|c|c|c|c|c|c|}
\hline $\mathbf{N}^{\circ}$ & $\mathbf{D i ́ a}$ & $\begin{array}{c}\mathbf{C} / \\
\mathbf{R S}^{\mathbf{a}}\end{array}$ & $\begin{array}{c}\mathbf{T i}_{\mathbf{a}}{ }^{\mathbf{b}} \\
\left({ }^{\circ} \mathbf{C}\right)\end{array}$ & $\begin{array}{c}\mathbf{T f}_{\mathbf{a}}{ }^{\mathbf{c}} \\
\left({ }^{\left.{ }^{\circ} \mathbf{C}\right)}\right.\end{array}$ & $\begin{array}{c}\mathbf{E}_{\mathbf{s}}{ }^{\mathbf{d}} \\
(\mathbf{M J})\end{array}$ & $\begin{array}{c}\mathbf{E}_{\mathbf{T}}{ }^{\mathbf{e}} \\
(\mathbf{M J})\end{array}$ & $\begin{array}{c}\mathbf{\eta}^{\mathbf{f}} \\
(\mathbf{\%})\end{array}$ \\
\hline 1 & $15 / 03 / 17$ & S & 22,00 & 43,14 & 18,39 & 8,78 & 47,74 \\
\hline 2 & $16 / 03 / 17$ & S & 23,07 & 49,13 & 21,73 & 10,81 & 49,76 \\
\hline 3 & $21 / 03 / 17$ & S & 26,72 & 52,84 & 20,98 & 10,82 & 51,56 \\
\hline 4 & $22 / 03 / 17$ & S & 24,89 & 51,56 & 18,63 & 11,05 & 59,31 \\
\hline 5 & $26 / 03 / 17$ & S & 24,29 & 48,95 & 19,90 & 10,23 & 51,40 \\
\hline 6 & $24 / 05 / 17$ & S & 21,12 & 50,62 & 18,54 & 12,24 & 66,01 \\
\hline 7 & $31 / 05 / 17$ & S & 23,20 & 49,20 & 17,68 & 10,79 & 61,02 \\
\hline 8 & $01 / 06 / 17$ & S & 23,60 & 47,20 & 16,20 & 9,79 & 60,43 \\
\hline 9 & $07 / 06 / 17$ & S & 22,79 & 50,70 & 18,49 & 11,57 & 62,85 \\
\hline 10 & $08 / 06 / 17$ & S & 23,22 & 52,01 & 19,00 & 11,93 & 62,82 \\
\hline 11 & $13 / 03 / 17$ & PN & 22,76 & 44,67 & 17,37 & 9,03 & 52,35 \\
\hline 12 & $20 / 03 / 17$ & PN & 22,69 & 49,13 & 21,87 & 10,97 & 50,14 \\
\hline 13 & $23 / 03 / 17$ & PN & 25,43 & 42,84 & 14,76 & 7,23 & 48,97 \\
\hline 14 & $25 / 03 / 17$ & PN & 23,75 & 42,61 & 15,18 & 7,83 & 51,61 \\
\hline 15 & $26 / 05 / 17$ & PN & 26,67 & 49,88 & 14,47 & 9,62 & 66,48 \\
\hline 16 & $30 / 05 / 17$ & PN & 23,22 & 42,69 & 13,37 & 8,09 & 60,46 \\
\hline 17 & $02 / 06 / 17$ & PN & 23,33 & 47,66 & 16,76 & 10,09 & 60,23 \\
\hline 18 & $03 / 06 / 17$ & PN & 24,01 & 47,89 & 15,95 & 9,91 & 62,12 \\
\hline 19 & $05 / 06 / 17$ & PN & 24,23 & 47,81 & 16,27 & 9,78 & 60,10 \\
\hline 20 & $06 / 06 / 17$ & PN & 22,79 & 45,76 & 16,14 & 9,53 & 59,07 \\
\hline 21 & $10 / 03 / 17$ & N & 23,57 & 31,77 & 5,37 & 3,41 & 63,53 \\
\hline 22 & $14 / 03 / 17$ & N & 25,00 & 35,12 & 7,33 & 4,21 & 57,48 \\
\hline 23 & $24 / 03 / 17$ & N & 23,96 & 39,33 & 11,08 & 6,39 & 57,66 \\
\hline
\end{tabular}

${ }^{\mathrm{a}}$ Condición de la radiación solar, ${ }^{\mathrm{b}}$ Temperatura inicial del agua del tanque de almacenamiento del CSTE, ${ }^{\mathrm{c}}$ Temperatura final del agua del tanque de almacenamiento del CSTE, ${ }^{\text {E Energía o }}$ calor térmica útil, ${ }^{\mathrm{e}}$ Energía solar incidente total, ${ }^{\mathrm{f}}$ Eficiencia.

Tabla 4. Eficiencia del CSTE con tubo de calor

\begin{tabular}{|c|c|c|c|c|c|c|c|}
\hline $\mathbf{N}^{\circ}$ & Día & $\begin{array}{c}\text { C/ } \\
\text { RS }^{\mathbf{a}}\end{array}$ & $\begin{array}{l}\mathrm{Ti}_{\mathrm{i}^{\mathrm{b}}} \\
\left({ }^{\circ} \mathrm{C}\right)\end{array}$ & $\begin{array}{l}\mathrm{Tf}_{\mathrm{a}}^{\mathrm{c}} \\
\left({ }^{\circ} \mathrm{C}\right)\end{array}$ & $\begin{array}{c}\mathrm{E}_{\mathrm{s}}{ }^{1} \\
(\mathrm{MJ})\end{array}$ & $\begin{array}{c}\mathbf{E}_{\mathrm{T}}^{\mathrm{e}} \\
(\mathrm{MJ})\end{array}$ & $\begin{array}{c}\eta^{f} \\
(\%)\end{array}$ \\
\hline 1 & $15 / 03 / 17$ & $\mathrm{~S}$ & 18,15 & 40,05 & 18,34 & 10,93 & 59,62 \\
\hline 2 & $16 / 03 / 17$ & $\mathrm{~S}$ & 21,62 & 42,69 & 21,66 & 10,50 & 48,50 \\
\hline 3 & $21 / 03 / 17$ & $\mathrm{~S}$ & 21,19 & 43,85 & 20,92 & 11,30 & 54,01 \\
\hline 4 & $22 / 03 / 17$ & $\mathrm{~S}$ & 22,00 & 42,85 & 18,58 & 10,89 & 58,63 \\
\hline 5 & $26 / 03 / 17$ & $\mathrm{~S}$ & 22,54 & 41,45 & 19,84 & 9,43 & 47,52 \\
\hline 6 & $24 / 05 / 17$ & $\mathrm{~S}$ & 21,34 & 45,76 & 18,49 & 12,16 & 65,81 \\
\hline 7 & $31 / 05 / 17$ & $\mathrm{~S}$ & 18,27 & 43,96 & 17,62 & 12,81 & 72,69 \\
\hline 8 & $01 / 06 / 17$ & $\mathrm{~S}$ & 18,72 & 39,71 & 16,15 & 10,48 & 64,90 \\
\hline 9 & $07 / 06 / 17$ & $\mathrm{~S}$ & 20,43 & 46,01 & 18,36 & 12,74 & 69,45 \\
\hline 10 & $08 / 06 / 17$ & $\mathrm{~S}$ & 21,42 & 45,12 & 18,94 & 11,81 & 62,38 \\
\hline 11 & $13 / 03 / 17$ & $\mathrm{PN}$ & 20,18 & 41,06 & 17,32 & 10,42 & 60,18 \\
\hline 12 & $20 / 03 / 17$ & $\mathrm{PN}$ & 17,97 & 43,25 & 21,81 & 12,61 & 57,82 \\
\hline 13 & $23 / 03 / 17$ & PN & 21,67 & 37,09 & 14,72 & 7,70 & 52,29 \\
\hline 14 & $25 / 03 / 17$ & PN & 21,55 & 37,31 & 15,13 & 7,83 & 52,01 \\
\hline 15 & $26 / 05 / 17$ & $\mathrm{PN}$ & 23,35 & 43,36 & 14,4 & 9,97 & 69,14 \\
\hline 16 & $30 / 05 / 17$ & PN & 20,61 & 38,72 & 13,33 & 9,04 & 67,70 \\
\hline 17 & $02 / 06 / 17$ & $\mathrm{PN}$ & 19,06 & 41,50 & 16,70 & 11,20 & 67,03 \\
\hline 18 & $03 / 06 / 17$ & $\mathrm{PN}$ & 21,47 & 42,05 & 15,90 & 10,26 & 64,57 \\
\hline 19 & $05 / 06 / 17$ & PN & 20,73 & 41,60 & 16,22 & 10,41 & 64,14 \\
\hline 20 & $06 / 06 / 17$ & $\mathrm{PN}$ & 21,95 & 40,41 & 16,09 & 9,20 & 57,21 \\
\hline 21 & $10 / 03 / 17$ & $\mathrm{~N}$ & 21,24 & 28,88 & 5,35 & 3,82 & 71,28 \\
\hline 22 & $14 / 03 / 17$ & $\mathrm{~N}$ & 22,51 & 30,80 & 7,30 & 4,14 & 56,72 \\
\hline 23 & $24 / 03 / 17$ & $\mathrm{~N}$ & 20,20 & 35,72 & 11,04 & 7,75 & 70,23 \\
\hline
\end{tabular}

${ }^{\mathrm{a}}$ Condición de la radiación solar, ${ }^{\mathrm{b}}$ Temperatura inicial del agua del tanque de almacenamiento del CSTE, ${ }^{\mathrm{c}}$ Temperatura final del agua del tanque de almacenamiento del CSTE, ${ }^{\text {d}}$ Energía o calor térmica útil, ${ }^{\mathrm{e}}$ Energía solar incidente total, ${ }^{\mathrm{f}}$ Eficiencia.
Finalmente, se presentan los promedios de las dos tablas anteriores de ambos colectores considerando las variables independientes.

Tabla 5. Promedios de las estimaciones respecto al CSTE sin y con tubo de calor y condición de la radiación solar

\begin{tabular}{|c|c|c|c|c|c|c|c|}
\hline $\mathbf{N}^{\circ}$ & $\mathrm{CS}^{\mathbf{a}}$ & $\begin{array}{c}\text { C/ } \\
\text { RS }^{\mathbf{b}}\end{array}$ & $\begin{array}{l}\mathrm{Ti}^{{ }^{\mathrm{c}}} \\
\left({ }^{\circ} \mathrm{C}^{2}\right)\end{array}$ & $\begin{array}{l}\mathbf{T f}_{\mathrm{a}^{\mathrm{d}}} \\
\left({ }^{\circ} \mathbf{C}\right)\end{array}$ & $\begin{array}{c}\mathbf{E}_{\mathrm{s}}{ }^{\mathrm{e}} \\
\text { (MJ) }\end{array}$ & $\begin{array}{c}\mathbf{E}_{\mathrm{T}}{ }^{\mathrm{f}} \\
(\mathbf{M J})\end{array}$ & $\begin{array}{c}\eta^{g} \\
(\%)\end{array}$ \\
\hline 1 & $\mathrm{~S} / \mathrm{TC}$ & $\mathrm{S}$ & 23,49 & 49,54 & 18,95 & 10,80 & 57,15 \\
\hline 2 & $\mathrm{~S} / \mathrm{TC}$ & PN & 23,89 & 46,09 & 16,21 & 9,21 & 57,29 \\
\hline 3 & $\mathrm{~S} / \mathrm{TC}$ & $\mathrm{N}$ & 24,18 & 35,41 & 7,93 & 4,67 & 59,56 \\
\hline 1 & $\mathrm{C} / \mathrm{TC}$ & $\mathrm{S}$ & 20,57 & 43,15 & 18,89 & 11,31 & 60,35 \\
\hline 2 & $\mathrm{C} / \mathrm{TC}$ & PN & 20,85 & 40,64 & 16,16 & 9,86 & 61,21 \\
\hline 3 & $\mathrm{C} / \mathrm{TC}$ & $\mathrm{N}$ & 21,32 & 31,80 & 7,90 & 5,24 & 66,08 \\
\hline
\end{tabular}

${ }^{\mathrm{a}}$ Colector solar, ${ }^{\mathrm{b}}$ Condición de la radiación solar, ${ }^{\mathrm{c}}$ Temperatura inicial del agua del tanque de almacenamiento del CSTE, d Temperatura final del agua del tanque de almacenamiento del CSTE, ${ }^{\mathrm{e}}$ Energía o calor térmica útil, ${ }^{\mathrm{f}}$ energía solar incidente total, ${ }^{\mathrm{g}}$ Eficiencia.

En la tabla anterior, para el CSTE sin tubo de calor, se observa que la eficiencia de días nublados es mayor en $2,41 \%$ y $2,27 \%$ respecto a días soleados y parcialmente nublados, respectivamente. De igual manera, para el CSTE con tubo de calor, se observa que la eficiencia de días nublados es mayor en 5,73 \% y 4,48 \% respecto a días soleados y parcialmente nublados, respectivamente. Esto se debe a que en días nublados existe una menor pérdida de calor por el menor incremento de temperatura en todas las partes del colector y un mayor porcentaje de aprovechamiento de la energía solar recibida.

En consecuencia, los CSTE con tubo de calor son ligeramente más eficientes por que aprovechan la energía solar incidente para cambiar de fase el alcohol contenido en el interior del tubo de calor de cobre, este vapor fluye hacia la parte superior del tubo donde se condensa, porque transfiere esa energía al agua que rodea el cabezal, esta transferencia es rápida por la alta conductividad térmica del cobre.

Por otro lado un CSTE sin tubo de calor es menos eficiente, porque la energía solar incidente calienta directamente el agua y lo único que se aprovecha es el calor sensible ganado, siendo esta trasferencia de calor más lenta.

\section{Proceso de prueba de hipótesis}

En el estudio se investigó el efecto de la condición de radiación solar y el tipo de CSTE en su eficiencia. Para estudiar el grado de relación entre los factores 
mencionados, se realizó un diseño experimental factorial de $2^{2}$.

La hipótesis para la investigación fue:

$\mathrm{H}_{0}=$ La condición de radiación solar y el tipo de colector solar influyen en la eficiencia de un colector solar de tubos evacuados.

$\mathrm{H}_{1}=$ La condición de radiación solar y el tipo de colector solar no influyen en la eficiencia de un colector solar de tubos evacuados.

El análisis de varianza se presenta a continuación:

Tabla 6. Análisis de varianza.

\begin{tabular}{|l|c|c|c|c|c|}
\hline \multicolumn{1}{|c|}{ Origen } & $\begin{array}{c}\text { Tipo III de } \\
\text { suma de } \\
\text { cuadrados }\end{array}$ & gl & $\begin{array}{c}\text { Cuadrático } \\
\text { promedio }\end{array}$ & F & Sig. \\
\hline Modelo corregido & $130,40^{\mathrm{a}}$ & 3 & 43,468 & 0,929 & 0,437 \\
\hline Interceptación & 139243,54 & 1 & 139243,5 & 2974,7 & 0,000 \\
\hline $\begin{array}{l}\text { Tipo de colector } \\
\text { solar }\end{array}$ & 126,629 & 1 & 126,629 & 2,705 & 0,109 \\
\hline $\begin{array}{l}\text { Condición de } \\
\text { radiación solar }\end{array}$ & 1,300 & 1 & 1,300 & 0,028 & 0,869 \\
\hline $\begin{array}{l}\text { Tipo de colector } \\
\text { solar * Condición } \\
\text { de radiación solar }\end{array}$ & 2,475 & 1 & 2,475 & 0,053 & 0,819 \\
\hline Error & 1685,13 & 36 & 46,809 & & \\
\hline Total & 141059,07 & 40 & & & \\
\hline Total corregido & 1815,533 & 39 & & & \\
\hline
\end{tabular}

Interpretación: Puesto que la significancia asintótica es grande (mayor que 0,05 ) en la variable condición de radiación solar, tipo de colector solar e interacción condición de radicación solar - tipo de colector solar, se concluye que no hay diferencias significativas en la eficiencia de un CSTE.

\section{Conclusiones}

Dos colectores solares de tubos evacuados con y sin tubo de calor fueron empleados para evaluar la eficiencia en condiciones reales de operación a diferentes condiciones de radiacion solar (días soleados, parcialmente nublados y nublados). El CSTE con tubo de calor comparado al CSTE sin tubo presentó ligeramente mayores eficiencias para las tres condiciones ambientales.

En los días nublados, existe una menor gradiente de temperatura entre el colector solar y el medio ambiente, las pérdidas de calor son menores y por lo tanto es mayor la eficiencia o el aprovechamiento de la energía solar incidente. Todo es contrario en los días soleados.
Estadísticamente, la condicion de radiacion solar, el tipo de colector y su interacción no influyen significativamente en la eficiencia de un CSTE. Debido a que la eficiencia varían en las experimentaciones porque es función del comportamiento de la radiación solar (aún mas en días parcialmente nublados), velocidad del viento, dia de la experimentación porque la radiacion varía durante el año, temperatura ambiente y ángulo de inclinación del colector solar.

\section{Recomendaciones}

Evaluar la influencia del ángulo de inclinación en la eficiencia del colector solar. En la literatura se indica que hay un efecto, pero se requiere cuantificarlo.

También es necesario evaluar la eficiencia del colector solar sin y con tubo de calor en otras épocas del año.

En las referencias, un CSTE tienen una superficies reflectante detrás de los tubos, por lo que se puede estudiar su efecto en la eficiencia.

\section{Referencias}

1. Ahmad, S. (2012). Modeling of flat-plate collector operation in transient states. Tesis maestría, Universidad de Purdue, Fort Wayne Indiana.

2. Amaris, J. M., Manrique, D. A., \& Jaramillo, J. E. (2015). Biocombustibles líquidos en Colombia y su impacto en motores de combustión interna. Una revisión. Revista Fuentes, 13(2), 23-34.

3. Ayala, F. E. B., Gómez, J. Q., \& León, E. A. (2011). Estudio de factibilidad del uso del biodiesel como fase contínua en lodos de perforación de emulsión inversa. Revista Fuentes, 9(1).

4. Budihardjo, I., \& Morrison, G. (2009). Performance of water-in-glass evacuated tube solar water heaters. Solar Energy, 83(1), 49-56. doi:10.1016/j.solener.2008.06.010

5. Conicet, I. (2009). Colectores solares para agua caliente. UNSA, Argentina.

6. Duffie, J., \& Beckman, W. (2013). Solar engineering of thermal processes (Cuarta ed.). New Jersey, Canada: Wiley.

7. Forero, L. E. P., Castro, Z. S., Bernal, H. R. G., \& Ávila, H. S. R. (2012). Hornillas paneleras Ward-Cimpa: validación de los modelos matemáticos de diseño Corpoica-UIS. Revista Fuentes, 10(2).

8. Hamed, M., Fellah, A., \& Ben Brahim, A. (2014). Parametric sensitivity studies on the performance of a flat plate solar collector in transient behavior. Energy Conversion and 
Management, 78, 938-947. doi:http://dx.doi. org/10.1016/j.enconman.2013.09.044

9. Jafarkazemi, F., \& Ahmadifard, E. (2013). Energetic and exergetic evaluation of flat plate solar collectors. Renewable Energy, 56, 55-63. doi:http://dx.doi.org/10.1016/j. renene.2012.10.031

10. Jesko, Z. (2008). Classification of solar collectors. Engineering for Rural Development, 22-27.

11. Kumar, A., \& Chandel, S. (2013). Tilt angle optimization to maximize incident solar radiation: A review. Renewable and Sustainable Energy Reviews, 23, 503-513. doi:http:// dx.doi.org/10.1016/j.rser.2013.02.027

12. Larrea, G., \& Bayas, H. (2011). Diseño de un sistema solar térmico para la producción de quesos en las comunidades: Chimborazo, Santa Isabel - Ecuador.

13. Loayza, F. (2012). Diseño e implementación de un seguidor solar para el control electrónico de un reflector Scheffler. Tesis título, Lima.

14. Martinez, J. C., Franco, P., \& Alonso, R. (2013). Manual termico de energía solar térmica. Uruguay.

15. Menjura, O. A. M., Tayo, J. L. N., Melo, E. R. V., Guerrero, A. C., \& Bernal, H. R. G. (2011). Ahorro de combustible y energía en horno s usados para la elaboración de panela modificando el diseño de pailas abiertas. Revista fuentes, 9(2).

16. Park, S., Pandey, A., Tyagi, V., \& Tyagi, S. (2014). Energy and exergy analysis of typical renewable energy systems. Renewable and Sustainable Energy Reviews, 30, 105-123. doi:http:// dx.doi.org/10.1016/j.rser.2013.09.011

17. Salcedo, C. (2011). Influencia del estudio de la tecnología de vacio en tubos sobre la eficiencia para el calentamiento de agua usando la energía solar. Tesis de grado, Universidad Técnica de Ambato.

18. Skeiker, K. (2009). Optimum tilt angle and orientation for solar collectors in Syria. Revista Energy Conversion and Management, 50, 2439 -2448. doi:10.1016/j.enconman.2009.05.031
19. Tagliafico, L., Scarpa, F., \& De Rosa, M. (2014). Dynamic thermal models and CFD analysis for flat-plate thermal solar collectors - A review. Renewable and Sustainable Energy Reviews, 30, 526-537. doi:http://dx.doi.org/10.1016/j. rser.2013.10.023

20. Taherian, H., Rezania, A., Sadeghi, S., \& Ganji, D. (2011). Experimental validation of dynamic simulation of the flat plate collector in a closed thermosyphon solar water heater. Energy Conversion and Management, 52, 301-307. doi:10.1016/j.enconman.2010.06.063

21. Vidal, E., \& Fontalvo, C. (2018). Alternativa para la generación de gas natural sintético a partir de una fuente de energía renovable mediante tecnología "Power to Gas" en Colombia. Revista Fuentes, 16(1), 71-79.

22. Zima, W., \& Dziewa, P. (2010). Mathematical modelling of heat transfer in liquid flatplate solar collector tubes. Archives of Thermodynamics, 31(2), 45-62.

\section{Simbología}

\begin{tabular}{|l|l|l|}
\hline Símbolo & \multicolumn{1}{|c|}{ Descripción } & Unidades \\
\hline $\mathrm{RS}$ & Radiación solar & $\mathrm{W} / \mathrm{m}^{2}$ \\
\hline $\mathrm{RSH}$ & Radiación solar que incide en la horizontal & $\mathrm{W} / \mathrm{m}^{2}$ \\
\hline$\eta$ & $\begin{array}{l}\text { Eficiencia energética del CSTE respecto a } \\
\text { radiación solar en la horizontal }\end{array}$ & $\%$ \\
\hline $\mathrm{T}_{\mathrm{a}}$ & $\begin{array}{l}\text { Temperatura promedio del agua de la parte } \\
\text { superior del tanque de almacenamiento }\end{array}$ & ${ }^{\circ} \mathrm{C}$ \\
\hline $\mathrm{T}_{\mathrm{ia}}$ & $\begin{array}{l}\text { Temperatura inicial del agua de la parte } \\
\text { superior del tanque de almacenamiento }\end{array}$ & ${ }^{\circ} \mathrm{C}$ \\
\hline $\mathrm{T}_{\mathrm{fa}}$ & $\begin{array}{l}\text { Temperatura final del agua de la parte } \\
\text { superior del tanque de almacenamiento }\end{array}$ & ${ }^{\circ} \mathrm{C}$ \\
\hline $\mathrm{E}_{\mathrm{S}}$ & Energía solar recibida & $\mathrm{MJ}$ \\
\hline $\mathrm{E}_{\mathrm{T}}$ & Energía o calor útil & $\mathrm{MJ}$ \\
\hline $\mathrm{S}$ & $\begin{array}{l}\text { Condición de la radiación solar de un día } \\
\text { soleado o claro }\end{array}$ & \\
\hline $\mathrm{N}$ & $\begin{array}{l}\text { Condición de la radiación solar de un día } \\
\text { nublado }\end{array}$ & $\begin{array}{l}\text { Condición de la radiación solar de un día } \\
\text { parcialmente nublado }\end{array}$ \\
\hline $\mathrm{PN}$ & & \\
\hline
\end{tabular}

Recepción: 03 de septiembre de 2018

Aceptación: 18 de marzo de 2019 